\title{
Short-term Unit Commitment with Simplified Economic Dispatch
}

\author{
C. L. CHEN and S. L. CHEN \\ Department of Electrical Engineering, National Taiwan University, Taipei, Taiwan (R.O.C.)
}

(Received September 13, 1990)

\section{ABSTRACT}

This paper is concerned with the solution of short-term thermal unit commitment problems. We follow the dynamic programming formulation of this problem which gives rise to a large-scale optimization problem. A truncated dynamic programming method incorporated with a simplified dispatch method is proposed. Programming techniques are described which minimize the execution time. Numerical experiments indicate that the proposed method finds a better solution in less time than many existing techniques.

\section{INTRODUCTION}

A complex problem that power utilities must encounter every day is to determine a schedule of units to meet the demand anticipated over a future 24 -hour period. It is widely recognized that a proper commitment of the available generating units is an important step in reducing costs during system operations. In general, the available approaches for unit commitment can be categorized into two groups. The first consists of rigorous optimization approaches that are impractical for application to problems of real size. The second consists of heuristic approaches that are actually used in practice, but give no assurance that the schedules produced are optimal or even close to optimal.

The unit commitment problem has been the subject of intensive research over past years. References 1-4 propose solution methods based on unit priority lists and heuristic rules to improve a given feasible solution. In refs. 5 and 6 , a solution methodology based on duality, Lagrangian relaxation, and nondifferentiable optimization is described. Merlin and Sandrin [7] propose another Lagrangian re- relaxation method; this is a decomposition method using Lagrange multipliers which provides a new solution to the conventional problem of thermal unit commitment. Dillion et al. [8] and Pang et al. [9] formulate the unit commitment problem as a linear mixed-integer programming problem and then use standard integer programming algorithms to solve for the commitment schedule. Several authors have used branch-and-bound methods [10 - 12] to solve this problem. Mucstadt and Koenig [13] propose using a branch-and-bound method along with Lagrangian relaxation to reduce the 'duality gap' in the Lagrangian relaxation approach.

The dynamic programming approaches [14 20] have been around the longest and are still widely used in power utilities. In the solution procedure of dynamic programming to unit commitment problems, a large number of unit combination states would consume an intolerable amount of computing time to find the optimal solution. It is noted by Lee [20] that, for a realistic utility system, the unit commitment cost function is nearly constant around the optimum and the accuracy of input data is somewhat uncertain. The nearly constant cost function often leads to high computational requirements in locating the theoretically 'optimal' solution. These characteristics suggest that a short-term unit commitment method should be judged by its ability to determine a 'near-optimal' solution with acceptable computational requirements. The usual remedy is to truncate the state space and reserve only some promising states for next-stage computations. Computational burdens could be reduced by decreasing the number of states to be computed. It should be noticed that a major portion of computing time is spent in performing the economic dispatch of each state. Calculation of the many economic dispatches for one day or a week is the main drain on 
computing time. If dispatch consumes less time, the computational burden would be relieved further and the commitment problem of large systems would be solvable.

\section{PROBLEM FORMULATION}

The objective of unit commitment is to schedule, with respect to the economics, available generating units under constraints. Costs are incurred both when starting up generating units and when dispatching them. The total cost over the study period is to be minimized. The problem variables are the hourly generating unit statuses, namely 'on' and 'off'.

Constraints can be divided naturally into two categories: the 'coupling' constraints and the 'local' constraints. The 'coupling' constraints, which concern the system of units overall, reflect the whole generation requirements, that is, the sum of the power generated by the units must be equal to or larger than the demand, including network losses, plus spinning reserve. The 'local' constraints deal with local operations at each thermal unit. The first constraint is the limit on the generated power; if the unit is started, its power must be within upper and lower generation limits. There are also technical constraints, together with the requirement of limiting equipment fatigue, leading to the imposition of minimum up times and minimum down times.

In mathematical terms, we state the unit commitment problem as

$\min \sum_{k=1}^{M} \sum_{i=1}^{N} C_{i}\left[P_{i}(k)\right]$

with constraints

$$
\begin{aligned}
& \sum_{i=1}^{N} P_{i}(k)=\operatorname{GR}(k) \quad k=1, \ldots, M \\
& I_{i, k} P \leqslant P_{i}(k) \leqslant I_{i, k} \bar{P}_{i} \\
& {\left[t_{\text {on }}(i, k-1)-T_{\text {on }}(i)\right]\left(I_{i, k-1}-I_{i, k}\right) \geqslant 0} \\
& {\left[t_{\text {off }}(i, k-1)-T_{\text {off }}(i)\right]\left(I_{i, k-1}-I_{i, k}\right) \geqslant 0}
\end{aligned}
$$

\section{SOLUTION METHODOLOGY}

\subsection{Dynamic programming}

Dynamic programming is a methodical procedure which systematically evaluates a large number of possible decisions in a multistep problem. The objective is to make a decision in each problem step which minimizes the total cost for all the decisions made. In our approach the computational structure of forward dynamic programming is adopted. There are practical reasons for going forward. The initial conditions are easily specified and the computations can go forward in time as long as required and as long as computer storage is available.

Before describing the procedure, the following four terms are defined.

A state is a combination of unit statuses. Given $N$ units, there are $2^{N}-1$ meaningful states altogether.

The search range denotes the restricted number of states to be searched at each stage.

A strategy denotes the transition from one state of a given stage to a state of the next stage. Such a transition may necessitate the starting up or shutting down of certain units.

A path is a combination of strategies from the initial stage to the present stage.

The outline of the approach is shown by the flowchart in Fig. 1. We arrange the unit order in strict order of priority, and the criterion for placing individual units at the various priority levels is the constant incremental production costs. In finding the feasible restricted search range for the next stage, we take a different approach from usual. The complete state selection list, which consists of $2^{N}-1$ states, is not formed in advance. We only generate the restricted search range which is feasible for the next stage. This process is rather simple. Upon creating the next-stage states, the higher-priority units are started up to increase the amount of generation, and the lower-priority units are shut down if the generation is to be decreased. In Fig. 2, the process of creating the next-stage search range is shown. States a, b, and c are stored with their least costs for stage $k$. Each of them then creates several feasible states for the $(k+1)$ th stage. After computing the fuel cost for every generated path to stage $k+1$. states e, f, and h with least costs in the search range of stage $k+1$ are saved. This process is repeated till the end.

Then we perform the dispatch of each state for the next stage. This procedure is described in the following Section. After the last stage is completed, the backtracking process is accompanied by lambda-iteration dispatches to get 


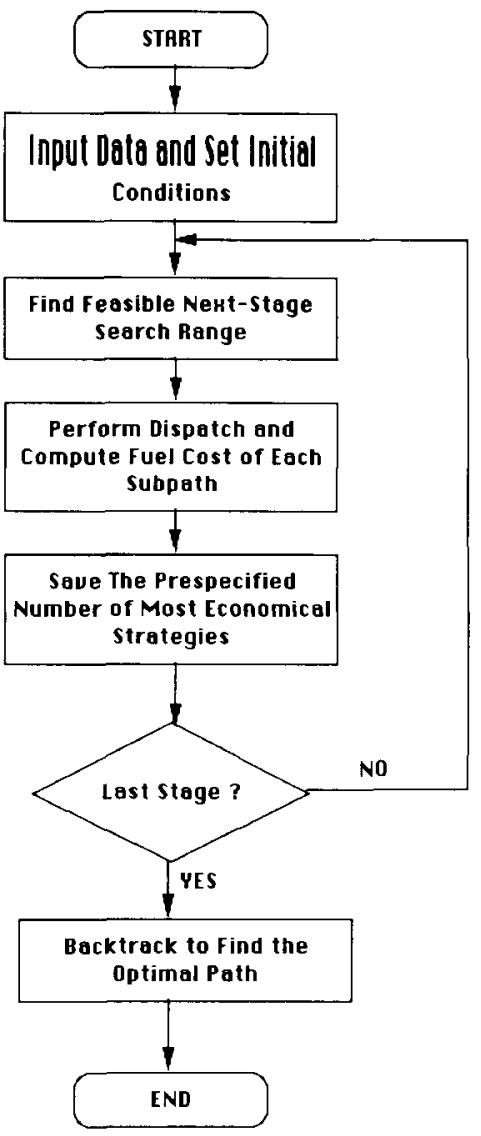

Fig. 1. Simplified flowchart of the unit commitment procedure.

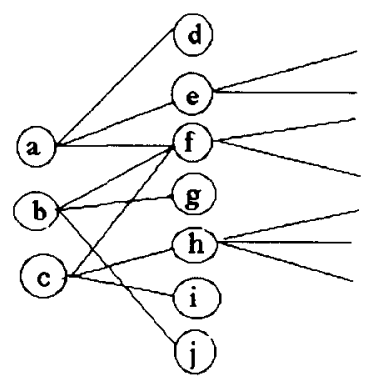

Stage $\mathrm{k} \quad$ Stage $\mathrm{k}+1$

Fig. 2. Creation of the next-stage search range.

the more accurate measures of the total fuel cost of the computed commitment path.

\subsection{Simplified dispatch}

In practical applications, a quadratic cost function is usually utilized to represent the unit production cost function. This technique uses economic dispatch methods [21] such as lambda iteration, first-order gradient, and second-order gradient methods which require it-

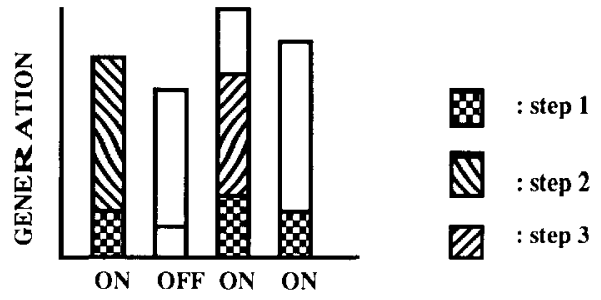

Fig. 3. A simple dispatch example.

erations or matrix inversions; these are the main drains on computing resources. In order to make these computations more efficient, a simplified model of the unit production cost function is used. A constant incremental cost rate between the minimum and maximum power points is assumed in our simplified method to compute the generation costs of each state. This constant incremental cost rate is derived by least-square approximation of $A+B P+C P^{2}$, the quadratic cost function usually adopted, to $K P$, where

$K=2(A C)^{1 / 2}+B$

A simple dispatch example of a four-unit system with commitment status $(1,0,1,1)$ is illustrated in Fig. 3. Because the generation of every running unit must fall between the upper and lower limits of its generation power, part of the load demand is first distributed to the lower generation limits of the running units. Then, according to the priority list of the running units, generation is increased to the upper limits of the units, one by one, until the generation requirement is met.

\section{NUMERICAL EXPERIMENTS}

This unit commitment procedure was tested on a 10-unit system to demonstrate its efficiency with respect to the length of time taken, and on a 20-unit system to compare it with a recently published method.

\subsection{The 10-unit system}

In the first example, a system with 10 generating units was studied. The unit data for system generation are given in Table 1 . The $K$ values are the computed constant incremental cost rates which are derived from $A, B$, and $C$. The generation requirements for each stage are shown in Table 2. Table 3 depicts the numerical results of our experiment. Compared with the lambda-iteration dispatch 
TABLE 1

Unit data for system generation

\begin{tabular}{rrrrrrrrrr}
\hline Unit & $\bar{P}$ & $P$ & STC & MU & MD & $A$ & $B$ & $C$ & $K$ \\
\hline 1 & 60 & 10 & 10 & 3 & 2 & 15 & 2.2034 & 0.0051 \\
2 & 80 & 20 & 12 & 3 & 5 & 25 & 1.9161 & 0.00396 & 2.75 \\
3 & 100 & 30 & 12 & 2 & 2 & 40 & 1.8518 & 0.00393 & 2.64 \\
4 & 120 & 25 & 13 & 3 & 2 & 32 & 1.6966 & 0.00382 & 2.40 \\
5 & 150 & 50 & 11 & 3 & 2 & 29 & 1.8015 & 0.00212 & 2.29 \\
6 & 280 & 75 & 18 & 6 & 6 & 72 & 1.5354 & 0.00261 & 2.40 \\
7 & 320 & 120 & 13 & 8 & 2 & 49 & 1.2643 & 0.00289 & 2.10 \\
8 & 445 & 125 & 15 & 10 & 5 & 82 & 1.2163 & 0.00148 & 1.91 \\
9 & 520 & 250 & 14 & 12 & 7 & 105 & 1.1954 & 0.00127 & 1.92 \\
10 & 550 & 250 & 20 & 12 & 3 & 100 & 1.1285 & 0.00135 & 1.86 \\
\hline
\end{tabular}

$\mathrm{STC}$, start-up cost; MU, MD, minimum up and down times; $A, B, C$, coefficients of quadratic cost function $A+B P+C P^{2}$; $K$, constant incremental cost rate; $\bar{P}, P$, upper and lower generation limits.

TABILE 2

Generation requirements

\begin{tabular}{llll}
\hline Hour & Gen. req. & Hour & Gen. req. \\
\hline 1 & 2000 & 13 & 1200 \\
2 & 1980 & 14 & 1160 \\
3 & 1940 & 15 & 1140 \\
4 & 1900 & 16 & 1160 \\
5 & 1840 & 17 & 1260 \\
6 & 1870 & 18 & 1380 \\
7 & 1820 & 19 & 1560 \\
8 & 1700 & 20 & 1700 \\
9 & 1510 & 21 & 1820 \\
10 & 1410 & 22 & 1900 \\
11 & 1320 & 23 & 1950 \\
12 & 1260 & 24 & 1990 \\
\hline
\end{tabular}

TABLE 3

Comparison of results of the lambda iteration and the proposed method

\begin{tabular}{lcc}
\hline & CPU time & Fuel cost \\
\hline $\begin{array}{l}\text { Lambda } \\
\text { iteration }\end{array}$ & 236 & 78950 \\
$\begin{array}{l}\text { Simplified } \\
\text { dispatch }\end{array}$ & 40 & 80227 \\
$\begin{array}{l}\text { Percentage } \\
\text { ratio }\end{array}$ & $16.9 \%$ & $101.6 \%$ \\
\hline
\end{tabular}

methods, only about $16.9 \%$ of computing time is required by this simplified dispatch method. However, a small increase in the total cost, about $1.6 \%$, accompanies the considerable saving in computation time.

\subsection{The 20-unit system}

In this example, the capability of the proposed method is illustrated in terms of its solution quality and computational requirements. A 20-unit midwestern system was used to compare the proposed method with Lee's successive iterative dynamic programming method [20]. The unit data and generation requirements for a 36 -hour commitment horizon can be acquired from Lee's paper [20]. The commitment schedule determined by the proposed method is shown in Table 4 and Table 5

TABLE 4

Proposed commitment schedule

\begin{tabular}{lllllllll}
\hline Hour & \multicolumn{2}{l}{ Unit } & \multicolumn{1}{l}{} \\
\cline { 2 - 8 } & $1-5$ & 6 & 7 & $8-9$ & 10 & 11 & 12 & $13-20$ \\
\hline $1-5$ & 1 & 0 & 1 & 0 & 0 & 0 & 0 & 0 \\
6 & 1 & 0 & 0 & 0 & 0 & 0 & 0 & 0 \\
$7-8$ & 1 & 0 & 0 & 0 & 0 & 0 & 1 & 0 \\
$9-12$ & 1 & 0 & 0 & 0 & 1 & 0 & 1 & 0 \\
$13-35$ & 1 & 0 & 0 & 0 & 1 & 0 & 0 & 0 \\
36 & 1 & 0 & 0 & 0 & 0 & 0 & 1 & 0 \\
\hline
\end{tabular}

TABLE 5

Comparison of results of Lee's method and the proposed method

\begin{tabular}{llc}
\hline & CPU time & Fuel cost \\
\hline Lee's method & 15 & 1053175 \\
Proposed method & 10.5 & 975077 \\
Percentage & $70 \%$ & $92.6 \%$ \\
ratio & & \\
\hline
\end{tabular}


gives the results of the comparison. It is worth noticing that only $70 \%$ of the CPU time of Lee's method is required on the same computer, a VAX-11/780, to produce a commitment schedule in which the total fuel cost is $92.6 \%$ of Lee's result.

\section{CONCLUDING REMARKS}

At present it is still not practical to determine the optimal commitment schedule, even for systems of reasonable size. Hence, most practical commitment procedures attempt to find the best schedule out of a set of schedules defined by some empirical or heuristic logic. The number of schedules in the defined set may be quite large, but is usually very small compared with the number of possible schedules. In order to evaluate many more schedules and consider the uncertainty of input data for computing the fuel cost, a simple and fast economic dispatch method is introduced in this paper. This method arranges running units in order of priority of the constant incremental cost rates and then distributes generation requirements sequentially. Without iterations, it consumes much less computing time than conventional dispatch methods. The need for such a fast and simple method of dispatch is evident if the number of economic dispatches that have to be performed to produce a unit commitment schedule is examined.

\section{NOMENCLATURE}

$C_{i}$ [ ] cost function of unit $i$

$\mathrm{GR}(k)$ generation requirement at stage $k$, which includes load demand, spinning reserve, and network losses

$I_{i, k} \quad$ status indicator of unit $i$ at stage $k$; with $I_{i, k}=0$, unit $i$ will be down at stage $k$, and with $I_{i, k}=1$, unit $i$ will be running at stage $k$

$M \quad$ number of stages

$N \quad$ number of units to be scheduled

$P_{i}(k) \quad$ generation of $i$ th unit at stage $k$

$\underline{P}_{i}, \bar{P}_{i} \quad$ lower and upper generation limits of unit $i$

$T_{\text {on }}(i)$ minimum up time of unit $i$

$T_{\text {off }}(i)$ minimum down time of unit $i$ $t_{\mathrm{on}}(i, k)$ time period that unit $i$ has been continuously up till stage $k$

$t_{\text {off }}(i, k)$ time period that unit $i$ has been continuously down till stage $k$

\section{REFERENCES}

1 R. M. Burns and C. A. Gibson, Optimization of priority lists for a unit commitment program, IEEE PES Sum mer Meeting, San Francisco, CA, U.S.A., 1975, Paper No. A 75 453-1.

2 L. T. Anstine, et al., Application of digital computer techniques to the economic scheduling of marginal generating units for the Pennsylvania- New Jersey. Maryland interconnection, IEEE Trans., PAS-83 (1964) 316 - 320.

3 M. E. El-Hawary and G. S. Christensen, Optimal Economic Operation of Electric Power Systems, Academic Press, New York, 1979.

4 R. R. Shoults, S. K. Chang, S. Helmick and W. M. Grandy, A practical approach to unit commitment, economic dispatch and savings allocation for multiplearea pool operation with import/export constraints, IEEE Trans., PAS-99 (1980) 625 - 635.

5 G. S. Lauer, D. P. Bertsekas, N. R. Sandell, Jr, and T. A. Posbergh, Solution of large-scale optimal unit com. mitment problems, IEEE Trans., PAS-101 (1982) 79. 86.

6 D. P. Bertsekas, G. S. Lauer, N. R. Sandell, Jr, and T. A. Posbergh, Optimal short-term scheduling of largescale power systems, IEEE Trans., AC-28 (1983) 1 - 11.

7 A. Merlin and P. Sandrin, A new method for unit commitment at Electricité de France, IEEE Trans., PAS-102 (1983) $1218-1225$.

8 T. S. Dillon, et al., Integer programming approach to the problem of optimal unit commitment with probabilistic reserve determination, IEEE Trans., PAS-97 (1978) 2154 - 2166.

9 C. K. Pang, et al., Pool daily fuel scheduling, EPRI Rep. No. EL-1659, Electric Power Research Institute, Palo Alto, CA, Feb. 1981.

10 A. I. Cohen and M. Yoshimura, A branch-and-bound algorithm for unit commitment, IEEE Trans., PAS-102 (1983) $444-451$.

11 A. Ohuchi and I. Kaji, A branch-and-bound algorithm for start-up and shut-down problem of thermal generating units, Electr. Eng. Jpn., 95 (1975) $54-61$.

12 B. Fox and S. P. Bond, Simple search technique for generating unit commitment, Int. J. Electr. Eng. Educ., 23 (1986) $263-271$.

13 J. A. Mucstadt and S. A. Koenig, An application of Lagrangian relaxation to scheduling in power generating systems, Oper. Res., 25 (1977) 387 - 403.

14 P. G. Lowery, Generating unit commitment by dynamic programming, IEEE Trans., PAS-85 (1966) $422-426$.

15 C. K. Pang and H. C. Chen, Optimal short-term thermal unit commitment, IEEE Trans., PAS-95 (1976) 1336 1346.

16 C. K. Pang, G. B. Sheble and F. Albuyeh, Evaluation of dynamic programming based methods and multiple area representation for thermal unit commitments, IEEE Trans., PAS-100 (1981) 1212 - 1218.

17 P. P. J. van den Bosch and G. Honderd, A solution of 
the unit commitment problem via decomposition and dynamic programming, IEEE Trans., PAS-104 (1985) $1684-1690$.

18 C. C. Nwannenna, A computer model for power system control, Comput. Oper. Res., 14 (1987) 325 - 340.

19 B. L. Synder, Jr, H. D. Powell, Jr and J. C. Rayburn,
Dynamic programming approach to unit commitment. IEEE Trans., PWRS-2 (1987) 339 - 350.

$20 \mathrm{~F}$. N. Lee, Short-term thermal unit commitment a new method, IEEE Trans., PWRS-3 (1988) 421 - 428.

21 A. J. Wood and B. F. Wollenberg, Power Generation Operation and Control. Wiley, New York, 1984, Ch. 3. 